\title{
Long-range Coulomb repulsion effect on a charged vortex in high-temperature superconductors with competing $d$-wave and antiferromagnetic orders
}

\author{
Hong-Wei Zhao, ${ }^{1}$ Guo-Qiao Zha, ${ }^{1}$ Shi-Ping Zhou,,${ }^{1,2}$ and F. M. Peeters ${ }^{2}$ \\ ${ }^{1}$ Department of Physics, Shanghai University, Shanghai 200436, China \\ ${ }^{2}$ Departement Fysica, Universiteit Antwerpen-Groenenborgerlaan 171, B-2020 Antwerpen, Belgium
}

(Received 9 July 2008; published 5 August 2008)

\begin{abstract}
Vortex charges in high-temperature superconductor (HTS) are studied by solving the Bogoliubov-de Gennes equations based on a model Hamiltonian with antiferromagnetic (AF) and $d$-wave orders in the presence of the long-range Coulomb repulsion. For a sufficient strength of the AF order, the negative vortex charge is found. A sign change between negative and positive may occur by tuning the long-range Coulomb repulsion strength or the doping parameter. Recent NMR experiments are hopefully understood. We show that the charged vortex can induce a spin-orbit coupling that is important for superconductors with a short coherence length and a large value of the energy gap over the Fermi-level ratio. Fractional flux quanta are possible for HTS.
\end{abstract}

DOI: 10.1103/PhysRevB.78.064505

PACS number(s): 74.20.-z, 74.25.Ha, 74.25.Jb

\section{INTRODUCTION}

The vortex charge in type-II superconductors has attracted considerable attention. ${ }^{1-9}$ In the framework of the BCS theory, one can show that for an $s$-wave superconductor the vortex charge is proportional to the derivative of the density of states at the Fermi level. In particular, by using the BCS expression for the transition temperature $T_{c}$, the sign of the vortex charge may be expressed as: $\operatorname{sgn}\left(\partial \ln T_{c} / \partial \mu\right)$. However, the results of the NMR measurements ${ }^{9}$ on slightly overdoped $\mathrm{YBa}_{2} \mathrm{Cu}_{3} \mathrm{O}_{7}$ (YBCO) and on underdoped $\mathrm{YBa}_{2} \mathrm{Cu}_{4} \mathrm{O}_{8}$ high-temperature superconductors (HTS) seemed, regarding the sign and the order of amplitude of the vortex charge, to be inconsistent with those predicted from the existing BCS theory. Chen et al. ${ }^{10}$ suggested that the competition of antiferromagnetic (AF) order with $d$-wave superconducting order (DSC) may vary the sign of the vortex charge. A negative vortex charge is predicted for the slightly overdoped sample, consistent with the NMR measurements. ${ }^{9}$ However, the problem remains unresolved for the underdoped case where the experiment ${ }^{9}$ tested a positively charged core, whereas the theory ${ }^{10}$ yielded the "electron-rich" one. In view of this discrepancy, together with the fact that vortex cores will charge up due to the Coulomb interaction between conducting electrons for superconductors having a small value of $k_{F} \xi$-as commonly observed in a neutral superfluid system, where $k_{F}$ is the Fermi wave vector and $\xi$ is the superconducting coherence length-we introduce the long-range Coulomb repulsion in the effective model Hamiltonian for HTS with competing DSC and spin-density wave (SDW) orders. ${ }^{11-15}$

We investigate the effect of the long-range Coulomb repulsion on the AF order and on the vortex charge. We show, via the fully self-consistent solution of the model, that the long-range Coulomb interaction competing with the AF order by expelling electrons out of the vortex core may lead to a sign change in the vortex charge from negative to positive even through the on-site repulsion $U$, that is assumed to respond for the AF-like spin-density wave order, remains large. The NMR experiments ${ }^{9}$ are, regarding both the vortex charge sign and the order of amplitude, hopefully understood when appropriate model parameters had been used. We emphasize that the charge accumulation around a vortex core is found to be $0.1 e$, which is $3-5$ orders larger than that for a typical type-II metallic superconductor. ${ }^{5,6}$ Such a large charge accumulation should lead to a strong electrostatic field that would generate a spin-orbit (SO) coupling. An additional geometric phase appears in connection with the SO term. Consequently, the measurable flux carried by the vortex is expected to be fractional (in units of the flux quanta $\Phi_{0}=h c / 2 e$ ) for HTS. This may shed light on the long-standing conjecture of timereversal symmetry breaking - a state that may relate to the fractional flux quanta ${ }^{16}$ and help our understanding about the high- $T_{c}$ superconductivity.

\section{MODEL}

We consider a two-dimensional lattice representing a YBCO superconductor with $M=M_{x} \times M_{y}$ magnetic units. Each magnetic unit has a linear dimension of $N=\left(N_{x} \times N_{y}\right)$. The effective Hamiltonian with competing AF and $d$-wave superconducting orders in the presence of a long-range Coulomb interaction is given by

$$
\begin{aligned}
H= & -\sum_{i, j, \sigma} t_{i j, \sigma} c_{i \sigma}^{\dagger} c_{j \sigma}+\sum_{i, \sigma}\left[U\left\langle n_{i \bar{\sigma}}\right\rangle-\mu\right] c_{i \sigma}^{\dagger} c_{i \sigma} \\
& +\sum_{\langle i, j\rangle}\left(\Delta_{i j} c_{i \uparrow}^{\dagger} c_{j \downarrow}^{\dagger}+\text { H.c. }\right)+\sum_{i, \sigma}\left(\frac{V_{c}}{2} \sum_{2<|l-i| \leq 5} \frac{n_{l}-\bar{n}}{\left|\vec{r}_{l}-\vec{r}_{i}\right|} c_{i \sigma}^{\dagger} c_{i \sigma}\right),
\end{aligned}
$$

where $c_{i \sigma}^{\dagger}\left(c_{i \sigma}\right)$ creates (annihilates) an electron of spin index $\sigma$ on the $i$ th site, $\mu$ is the chemical potential, and $U$ is the on-site repulsion that is assumed to generate an AF-like SDW order $\Delta_{\mathrm{SDW}}=U\left\langle c_{i \uparrow}^{\dagger} c_{i \uparrow}-c_{i \downarrow}^{\dagger} c_{i \downarrow}\right\rangle$. The sum over $i, j$ in the hopping term includes both the nearest-neighboring and next-nearest-neighboring (NNN) sites, whereas the pairing attraction involves only the nearest-neighboring ones. As to the long-range Coulomb term, the sum over $l$ runs up to its fifth-nearest neighboring for each $i$ th site within the core regime to account for the so-called Thomas-Fermi (TF) screening effect.

The $(M \times N)$ dimensional problem can be reduced into the $N$-dimensional one. Let us denote the translation vector be- 
tween two magnetic units as $\vec{R}=\left(m N_{x} a, n N_{y} a\right)$. Translation operation on the pairing potential $\Delta$ and the quasiparticle wave functions $u_{\vec{k}, \sigma}$ and $\nu_{\vec{k}, \sigma}$ gives

$$
\mathcal{T}[\Delta(\vec{r})] \equiv \Delta(\vec{r}+\vec{R}) \rightarrow e^{i \chi(r, R)} \Delta(\vec{r})
$$

and

$$
\left(u_{\vec{k}, \sigma}, v_{\vec{k}, \sigma}\right)_{\vec{r}+\vec{R}} \rightarrow e^{i \vec{k} \cdot \vec{R}}\left[e^{i \chi / 2} u_{\vec{k}, \sigma}(\vec{r}), e^{-i \chi / 2} v_{\vec{k}, \sigma}(\vec{r})\right]
$$

with the phase accumulation $\chi(\vec{r}, \vec{R})=2 \pi \vec{A}(\vec{r}) \cdot \vec{R} / \Phi_{0}-4 m n \pi$. This sets the self-consistent and the periodic boundary conditions for $\Delta, u_{\sigma}^{\alpha}(k)$, and $v_{\sigma}^{\alpha}(k)$ that reduce the initial problem to an $N$-dimensional one. ${ }^{17}$ We then diagonalize Hamiltonian (1) by solving the Bogoliubov-de Gennes (BdG) Equation (2) within one magnetic unit.

$$
\sum_{j}\left(\begin{array}{cc}
H_{i j, \sigma} & \Delta_{i j} \\
\Delta_{i j}^{*} & -H_{i j, \sigma}^{*}
\end{array}\right)\left(\begin{array}{c}
u_{j, \sigma}^{n} \\
v_{j, \sigma}^{n}
\end{array}\right)=\varepsilon_{n}\left(\begin{array}{c}
u_{i, \sigma}^{n} \\
v_{i, \sigma}^{n}
\end{array}\right),
$$

where

$$
\begin{aligned}
H_{i j, \sigma}= & -t_{i j}+\frac{V_{c}}{2}\left\langle c_{j, \sigma}^{\dagger} c_{i, \sigma}\right\rangle \\
& +\left[U\left\langle n_{i, \bar{\sigma}}\right\rangle-\mu+\frac{V_{c}}{2} \sum_{l \neq i}\left(\left\langle n_{l}\right\rangle-\bar{n}\right) / r_{i, l}\right] \delta_{i, j} .
\end{aligned}
$$

The self-consistent conditions are given by: $\Delta_{i j}$ $=\Sigma_{n}\left\{\frac{V_{d}}{4}\left(u_{i}^{n} v_{j}^{n^{*}}+v_{i}^{n^{*}} u_{j}^{n}\right) \tanh \left(\frac{\beta \varepsilon_{n}}{2}\right)-\frac{V_{c}}{2} v_{i}^{n^{*}} u_{j}^{n}\left[1-f\left(\varepsilon_{n}\right)\right]\right\}, \quad$ and $\left\langle n_{i \uparrow}\right\rangle=\Sigma_{n}\left|u_{i}^{n}\right|^{2} f\left(\varepsilon_{n}\right)$ and $\left\langle n_{i \downarrow}\right\rangle=\Sigma_{n}\left|v_{i}^{n}\right|^{2}\left[1-f\left(\varepsilon_{n}\right)\right]$ with the wave-function vectors denoted as $u_{i}^{n}=\left(u_{i, \uparrow}^{n},-v_{i, \uparrow}^{n *}\right)$ and $v_{i}^{n}$ $=\left(v_{i, \downarrow}^{n}, u_{i, \downarrow}^{n *}\right)$, where $f(E)=1 /\left(e^{\beta E}+1\right)$ is the Fermi distribution function. $V_{d}$ is the coupling strength. The DSC order parameter is defined as $\Delta_{i}^{D}=\left(\Delta_{i+e_{x},}^{D}+\Delta_{i-e_{x} i}^{D}-\Delta_{i, i+e_{y}}^{D}-\Delta_{i, i-e_{y}}^{D}\right) / 4$ with $\Delta_{i j}^{D}=\Delta_{i j} \exp \left[i \frac{\pi}{\Phi_{0}} \int_{\vec{r}_{i}}^{\left(\vec{r}_{i}+\vec{r}_{j}\right) / 2} \vec{A} \cdot d \vec{r}\right]$ and $e_{x, y}$ the unit vector along the $(x, y)$ direction. The vortex charge density (in units of $-|e|)$ is $n_{i}=\left\langle c_{i \uparrow}^{\dagger} c_{i \uparrow}+c_{i \downarrow}^{\dagger} c_{i \downarrow}\right\rangle=\left\langle n_{i \uparrow}\right\rangle+\left\langle n_{i \downarrow}\right\rangle$. The averaged charge density is $\bar{n}=\sum_{i \sigma}\left\langle c_{i \sigma}^{\dagger} c_{i \sigma}\right\rangle / N$.

In our calculations, the distance is measured in units of the lattice constant $a$ and the energy in the nearest-neighbor hopping integral parameter $t_{0}$. We took the values of $U$ from 1.5 up to 6 such that a reasonable description to the lowlying quasiparticle states can be expected. ${ }^{18}$ In addition, unlike the extreme limits of $U / V_{d} \gg 1$ or $U / V_{d} \ll 1$ where either a pure DSC or the SDW states are present, the coexistence of the SDW and DSC orders is expected for the intermediate value of $U / V_{d}$. For the concentration, we set $V_{d}=1.25$ and the ratio of $U$ to $V_{d}$ varies in the range of (1.2-2.0). In the presence of a magnetic field $\vec{h}=\nabla \times \vec{A}$ the hopping integral can be expressed as $t_{i j}=\bar{t} \exp \left[i \frac{e}{\hbar c} \int_{\vec{r}_{j}}^{\vec{r}_{i}} \vec{A} \cdot d \vec{r}\right]$. The average field was estimated as $B \sim 25 \mathrm{~T}\left(\gg H_{c 1}\right)$, assuming each magnetic unit of dimension $N_{x} \times N_{y}=48 \times 24$ contains two superconducting flux quanta $\Phi_{0}=h c / 2 e$. We neglect the supercurrent effect and choose a symmetric gauge $\vec{A}=\frac{1}{2}(-B y, B x, 0)$. The next-nearest-neighbor hopping $t^{\prime}=-0.20$ was selected to yield a reasonable magnitude of the DSC order parameter. We report on the results for $V_{c}=e^{2} /\left(2 \varepsilon_{e} a^{2}\right)$ in the range of $(0 \sim 0.35)$, which corresponds to a static dielectric constant from $\varepsilon_{e} \rightarrow \infty$ to $\varepsilon_{e} \sim 15 .{ }^{19}$
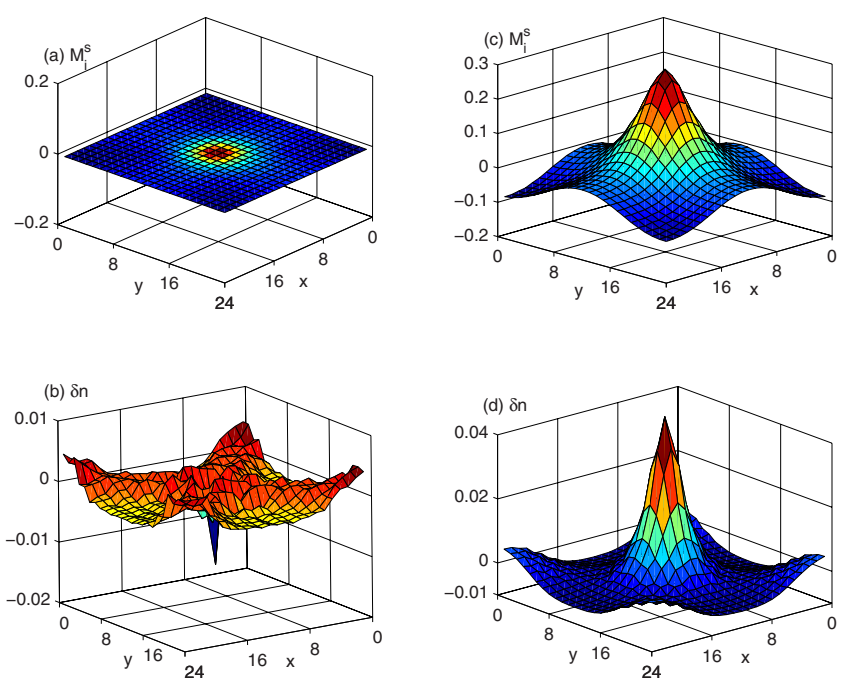

FIG. 1. (Color online) Spatial variations of the staggered magnetization $M_{i}^{s}$ and the relative electron density $\delta n_{i}=n_{i}-\bar{n}$ in a 24 $\times 24$ lattice. The left panels [(a) and (b)] and the right panels [(c) and (d)] are for $U=1.6$ and $U=2.2$, respectively. The average electron density $\bar{n}=0.82$.

\section{RESULTS AND DISCUSSIONS}

We first discuss the effect of the on-site repulsion $U$ without including the long-range Coulomb interaction. For small $U(=1.6)$ the staggered magnetization $M_{i}=(-1)^{i}\left\langle n_{i \uparrow}-n_{i \downarrow}\right\rangle$ is found in the order of $10^{-3}$ [Fig. 1(a)] and a pure DSC order with fourfold symmetry (not shown) has been obtained. The vortex core is positively charged, as shown in Fig. 1(b). In this case, the HTS may be treated as a system with two electronic subsystems: One of which is superconducting and the other with the core size in the order of coherence length remains normal. A chemical-potential difference is present between these subsystems, which drives the electrons outside from the core, resulting in a positively charged core. On the contrary, for a large $U(=2.2)$ the $\mathrm{AF}$ order inside the vortex core becomes significant [Fig. 1(c)], which-competing with the DSC order through expulsion of the holes from outside the vortex core-leads to a negative vortex charge [Fig. 1(d)]. Hence, the vortex core will be negatively charged for a slightly overdoped HTS where the AF and DSC orders coexist, ${ }^{20}$ whereas positive core charges are expected for highly overdoped samples where the AF order vanishes (inconsistent with the experiments and previous theoretical works).

We now investigate the influence of the long-range Coulomb interaction on the vortex charge. We consider an AFlike core by fixing the on-site repulsion strength $U=2.2$. An inclusion of the long-range Coulomb repulsion seems to weaken the on-site repulsion effect. As shown in the expression below [Eq. (2)] an opposite sign, associated with these two contributions to the Hartree term, is expected for an AF-like core with a local charge density $n_{l}$ less than the average density $\bar{n}$. Qualitatively, the Coulomb interaction would lead to somewhat a decline in the chemical potential of an electronic system. This may be equivalent to an increase in the doping concentration. The AF order then de- 

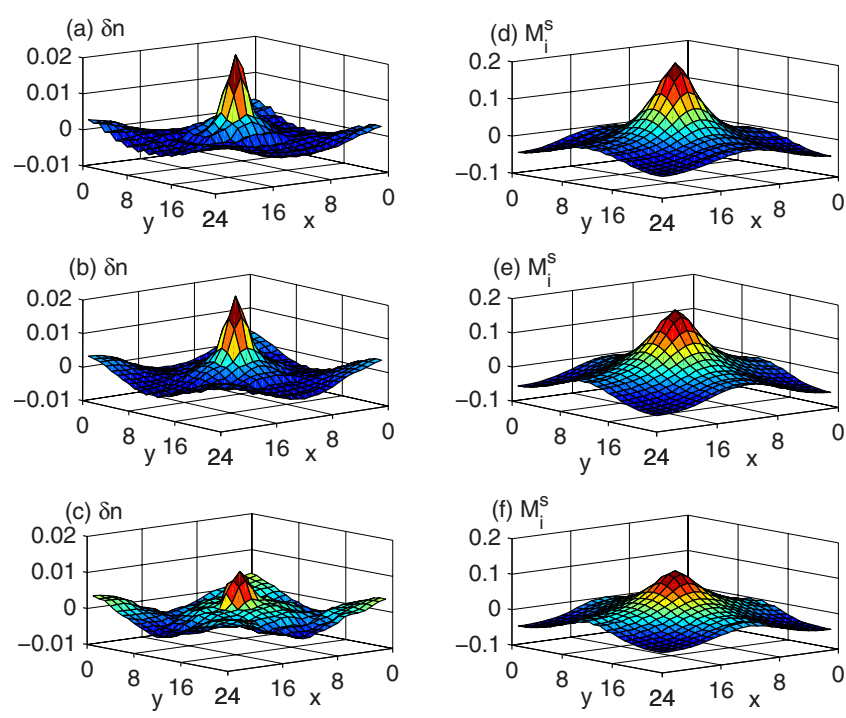

FIG. 2. (Color online) The net vortex charge density $\delta n_{i}=n_{i}$ $-\bar{n}$ and the staggered magnetization for the Coulomb expulsion strength $V_{c}=0$ [(a) and (d)], $0.15[(\mathrm{~b})$ and (e)], and 0.25 [(c) and (f)], respectively. The averaged electron density is $\bar{n}=0.865$.

creases, as inferred from the phase diagram of the HTS system. Indeed, the amplitude of both the core electron density [Figs. 2(a)-2(c)] and the staggered magnetization [Figs. 2(d)-2(f)] or the SDW drops gradually with an increase in $V_{c}$ accompanied with sharp spatial variations moving forward inside the core regime. We believe that it could be correlated with the destructive interference effect in which the moment of the spin induced by the on-site repulsion is screened by the spins of the surrounding quasiparticles. ${ }^{21,22}$ This is the analog to the vortex configuration transition from a stripe to the checkerboard pattern by increasing the NNN hopping strength. Actually, the resulting checkerboard pattern of the charge ordering textures can be seen as a lattice of ferromagnetic polarons or the "ferros" that reside at the intersections of a crossed array of antiphase domain walls of the AF order parameter. An increase in the NNN hopping strength seemed to break more AF bonds and to have larger ferromagnetic islands to accommodate the holes. ${ }^{23,24}$ With further increase in Coulomb repulsion strength up to 0.335 , the positively charged core is observed [Fig. 3(b)] even though the staggered magnetization or the core AF order remains large [Fig. 3(a)]. We noticed that the DSC order magnitude (not shown) is less than that for $V_{c}=0$, as expected. These calculations indicate that the vortex charge sign change may occur by tuning the long-range Coulomb interaction strength. It is important to realize that the effective static dielectric constant increases with hole concentration $\delta$ in HTS. ${ }^{19}$ One can then select a large value of $V_{c}$ for the underdoped sample, but a small one for the overdoped one. Therefore, a sign change from positive to negative is expected as the doping level increases from the underdoped to the slightly overdoped regime, while the on-site repulsion strength is kept constant. Consequently, NMR experiments ${ }^{9}$ can be understood by including the long-range Coulomb repulsion in the mean-field model for a HTS with competing AF and DSC orders.

The charge accumulation around a vortex core was found to be $0.1 e$ by calculating an integral over the core regime as presented in Fig. 1(d), which (in the same order with the experimental data $)^{9}$ is several orders larger than that for a typical type-II metallic superconductor. ${ }^{5,6}$ This can be understood from basic physics arguments below. (Since there is a lack of a common consensus about the microscopic theory for high- $T_{c}$ superconductivity, we presented our phenomenological arguments, as one usually does for a metallic superconductor.)

Consider the Sommerfeld free-electron model for a metal. Temperature dependence of carrier density $\rho$ at fixed chemical potential $\mu$ can be expressed $\operatorname{as}^{25} \rho(T) \approx n(0)$ $\left[1+\left(\pi^{2} / 8\right)\left(K_{B} T\right)^{2} / \mu^{2}\right]$. The carrier density increase due to a finite temperature smearing the Fermi level is estimated in the order of $\delta \rho \approx\left(\pi K_{B} T\right)^{2} \partial N(\varepsilon) /\left.\partial \varepsilon\right|_{\mu} / 3$. The transition of a system to superconducting state or the opening of a gap $\Delta$ in the spectrum yields an analogous smearing in the occupation probability, leading to the density increase varying with $\Delta$ as $\delta n \approx \Delta^{2} d N(E) / d E \ln \left(\hbar \omega_{D} / K_{B} T_{C}\right)$. Therefore, the chemical potential increase reads,

$$
\begin{aligned}
\delta \mu(\vec{r}) & =1 /\left.N\left(\varepsilon_{F}\right)(\partial N / \partial E)\right|_{\varepsilon_{F}} \ln \left(\hbar \omega_{D} / K_{B} T_{C}\right) \Delta^{2}(\vec{r}) \\
& =\left(d \ln K_{B} T_{C} / d \mu\right) \Delta^{2}(r) .
\end{aligned}
$$

[We have used the BCS expression $K_{B} T_{C} \approx \hbar \omega_{D} \exp$ $\left[-1 / N\left(\varepsilon_{F}\right) V\right]$. $]$ For a superconductor in the clean limit, we arrive at $\delta \mu=\left(\hbar^{2} / m_{e} \xi_{0}^{2}\right)\left(2 / \pi^{2} \Delta_{0}^{2}\right)\left(d \ln K_{B} T_{c} / d \ln \tilde{\mu}\right) \Delta^{2}(\vec{r})$, where $\tilde{\mu}$ and $\Delta_{0}$ are the Fermi level and the energy gap at zero temperature, respectively, and $m_{e}$ is the electron mass. It is then seen that the vortex charge effect would be enhanced by 3-5 orders of magnitude over the conventional BCS superconductor due to a short coherence length and a large value of the energy gap over the Fermi-level ratio in HTS.
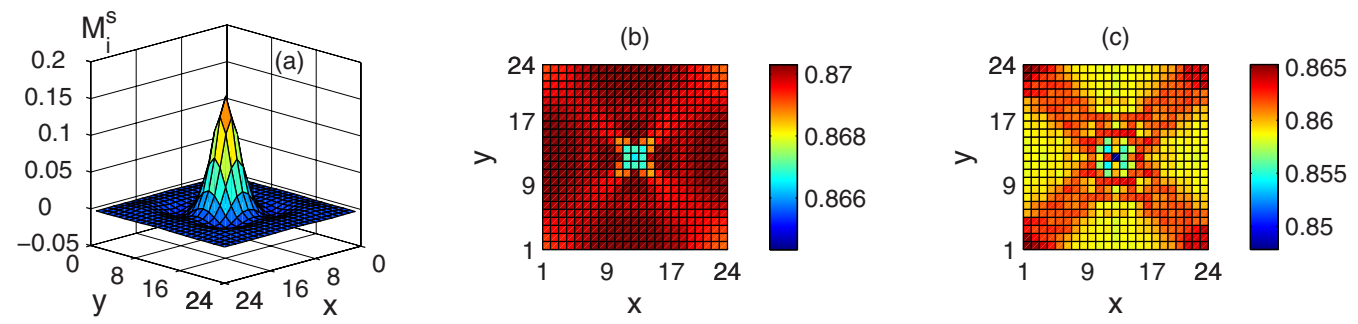

FIG. 3. (Color online) (a) Spatial variation of the staggered magnetization $M_{i}^{s}$ and (b) the contour plot of the vortex charge density for the Coulomb expulsion strength $V_{c}=0.335$, in resemblance with that for $U=1.6$ (c). A sign change in the core charge occurs in comparison with those for weak Coulomb interaction strengths shown in Fig. 2. 
Such a large charge accumulation should lead to a strong electrostatic field that would generate a SO coupling term. An additional geometric phase appears in connection with the SO term. Consequently, the measurable flux carried by a vortex is expected to be fractional. This may shed light on the long-standing conjecture of time-reversal symmetry breaking - a state that may lead to the fractional flux quanta in HTS. We address this issue below.

Charged vortices will induce an electrostatic electric field that provides the necessary force for the circular motion of quasiparticles and for a stable vortex structure. This field acts on the quasiparticle of spin $\vec{\sigma}$, mass $m$, and charge $e$ adding a SO coupling $\hat{H}_{\text {so }}$ to the Hamiltonian of the system. ${ }^{26} \mathrm{We}$ assume only the radial component of the electrostatic field $E_{r}$ and the azimuthal component of the vector potential $A_{\theta}$. Thereby the third component of spin-orbit interaction is necessarily to be included. This assumption is justified since our model, together with the numerical results, is symmetry under rotation. The $\mathrm{SO}$ term reduces to $H_{\text {so }}=-\left(e \hbar / 4 m^{2} c^{2} \sigma_{z}\right) \cdot \vec{E}_{r}(-i \hbar \nabla-e \vec{A} / c)_{\theta}$, with $\sigma_{z}=\left(\begin{array}{cc}1 & 0 \\ 0 & -1\end{array}\right)$. Equation (2) will then be broken up into two independent matrix equations due to the spin-orbit interaction: One is for the spin-up electron and spin-down hole wave function $\hat{\psi}_{i, \uparrow}$ $\equiv\left(u_{i, \uparrow}, v_{i, \downarrow}\right)$ and the other $\hat{\psi}_{i, \downarrow} \equiv\left(u_{i, \downarrow}, v_{i, \uparrow}\right)$ is for the spindown electron and spin-up hole. Each matrix equation has the same form, and we consider the $\hat{\psi}_{i, \uparrow}$. We can prove ${ }^{26}$ that the total magnetic flux $\Delta \chi$ that is quantized in units of the superconducting flux quanta reads

$$
\begin{aligned}
\Delta \chi & =\oint_{\text {u.c. boundary }} \vec{A} \cdot d \vec{l}+\left(\mu_{q} / e\right) \oint_{\text {u.c. boundary }}\left(\vec{E}_{r} \times d \vec{l}\right) \cdot \hat{z} \\
& \equiv \Phi(r)+\Phi_{\mathrm{SO}}(r)
\end{aligned}
$$

where $\Phi(r)$ measures the magnetic flux penetrating into the magnetic unit cell (u.c.), whereas

$$
\begin{aligned}
\Phi_{\mathrm{SO}}(r) & \equiv\left(\mu_{q} / e\right) \oint_{\text {u.c. boundary }}\left(\vec{E}_{r} \times d \vec{l}\right) \cdot \hat{z} \\
& =\left(\mu_{q} / e\right) \iint_{\text {u.c. area }} \nabla \cdot \vec{E}_{r} d s,
\end{aligned}
$$

is the flux due to the SO interaction, where $\mu_{q}=e \hbar / 4 m c$. Therefore, the AB-like magnetic flux $\Phi(\vec{r})$ is quantized when the SO flux $\Phi_{\text {so }}$ is negligible, whereas a sufficiently large contribution from the SO interaction would lead to a fractional or noninteger magnetic flux $\Phi(\vec{r})$. Substituting Poisson's equation $\nabla \cdot \vec{E}=-4 \pi \rho$ into Eq. (3b), we obtain

$$
\Phi_{\mathrm{SO}}=2 \cdot \sum_{k} a_{0} \int[n(\vec{r} ; \vec{k})-\bar{n}] d x d y\left(e^{2} / \hbar c\right)^{2},
$$

in units of $\Phi_{0}$, where $a_{0}$ is the Bohr radius and $\left(e^{2} / h c\right)$ $=1 / 137$. The prefactor of two in expression (4) accounts for contributions from both $\hat{\psi}_{i, \uparrow}$ and $\hat{\psi}_{i, \downarrow}$ states. Performing the integral on the charge distribution in Fig. 1(d) and summing over possible quasiparticle states of the wave vector $k$
$=1,2, \ldots$ and $M=24 \times 48$, we obtain $\Phi_{\text {so }} \approx 0.105 \pm 2 \times 10^{-3}$, indicating a noninteger AB-like flux quanta.

We want to stress that vortex charging is general and should occur in any type-II superconductor in the mixed state. Our prediction is, however, not in conflict with the flux quantization in metallic superconductors. We have shown that the vortex field magnitude is approximately of the order of $\left(\Delta^{2} / \xi_{0} \varepsilon_{F}\right) .^{27}$ Thus, the charged vortex effect is-in particular-important in those materials having relatively large value of $\Delta^{2} / \xi_{0} \varepsilon_{F}$, e.g., the HTS. Indeed, the core charge accumulation is calculated in the order of $10^{-1} e$, which is 3-5 orders larger than that for a typical metallic superconductor. ${ }^{6}$ It follows from the above discussion that the spin-orbit flux may be negligible for metallic superconductors rather than for high-temperature superconductors. Although an exclusive conclusion needs further experimental verification, the experiments that demonstrate the presence of fractional vortices in $d$-wave superconductors seem to support our arguments. ${ }^{28}$

We present a brief discussion about the experimental observation of the charged vortex effect. We suggest using the ferromagnet/superconductor structure ${ }^{29}$ to probe the spinHall effect ${ }^{30}$ on a nonmagnetic impurity doped HTS strip Hall cross with its one branch in contact with a ferromagnetic electrode. As temperature decreases below $T_{c}$, the impurity site captures a vortex core that may have a very sharp charge profile. When a spin current is injected out of the ferromagnetic electrode into one superconducting strip, the vortex charge induced spin-orbit interaction may lead to spin-down and spin-up separations. The polarized spins then flow in both superconducting branches in opposite directions under the action of the local electrochemical potential, resulting in an observable spin-Hall voltage.

\section{CONCLUSIONS}

In summary, starting with a model Hamiltonian with competing AF, $d$-wave superconducting orders, and long-range Coulomb interaction for HTS, we show that the superconducting condensate leads essentially to a positive vortex charge while the AF spin-density wave order favors to a negatively charged core. The long-range Coulomb interaction shows a mechanism for electron depletion inside the AF-like vortex core by breaking the AF bonds and by generating the hole-rich ferros. A first-order transition from negatively to positively charged vortices may occur by increasing the long-range Coulomb repulsion strength even though the core AF order remains intuitive. The NMR measurements ${ }^{9}$ are well understood. We demonstrated also that fractional $\mathrm{AB}$ flux quanta are expected for HTS where the spin-orbit flux becomes significant because of a short coherence length and the large value of the superconducting gap over the Fermi-level ratio.

The long-range Coulomb interaction is important as underdoped, or even slightly overdoped HTS materials are "bad" metals. (Only the overdoped material may be treated as a metal.) Numerous experimental observations such as angular resolved photoemission spectra, NMR measurements, scanning tunneling spectra, etc. provide strong evi- 
dences. Furthermore, on theoretical principles, Fetter (Ref. 31) investigated the screening effect in the metallic superconductors and showed that Thomas-Fermi (TF)-type screening is dominant. As is well known, however, the TF approximation is valid only in the long-wavelength limit since the short wavelength part of the Coulomb interaction between conduction electrons that has been shown to be more crucial for superconductors having a short coherence length is completely neglected. Actually, Blatter et al. ${ }^{5}$ discussed the screening effect with the TF approximation. They found that it reduces the vortex charge by a factor of $\left(\lambda_{\mathrm{TF}} / \xi\right)^{2}$, where $\lambda_{\mathrm{TF}}$ and $\xi$ are the Thomas-Fermi screening length and super- conducting coherence length, respectively. It is then seen that the TF screening effect would significantly reduce for a HTS with a very short coherence length.

\section{ACKNOWLEDGMENTS}

This work was supported by the National Natural Science Foundation of China under Grant No. 60671042 and by Science and Technology Committee of Shanghai Municipal under Grant No. 06JC14032. The authors (S.P.Z. and F.M.P.) acknowledge the financial support from the Flemish Science Foundation (FWO-Vl) and the Belgian Science Policy (IAP).
${ }^{1}$ D. I. Khomskii and A. Freimuth, Phys. Rev. Lett. 75, 1384 (1995).

${ }^{2}$ Hong-Yi Chen and C. S. Ting, Phys. Rev. B 71, 220510(R) (2005).

${ }^{3}$ D. Knapp, C. Kallin, A. Ghosal, and S. Mansour, Phys. Rev. B 71, 064504 (2005).

${ }^{4}$ C. Berthod, Phys. Rev. B 71, 134513 (2005).

${ }^{5}$ G. Blatter, M. Feigel'man, V. Geshkenbein, A. Larkin, and A. van Otterlo, Phys. Rev. Lett. 77, 566 (1996).

${ }^{6}$ J. Kolacek, P. Lipavsky, and E. H. Brandt, Phys. Rev. Lett. 86, 312 (2001)

${ }^{7}$ M. Machida and T. Koyama, Phys. Rev. Lett. 90, 077003 (2003).

${ }^{8}$ T. Nagaoka, Y. Matsuda, H. Obara, A. Sawa, T. Terashima, I. Chong, M. Takano, and M. Suzuki, Phys. Rev. Lett. 80, 3594 (1998).

${ }^{9}$ K. I. Kumagai, K. Nozaki, and Y. Matsuda, Phys. Rev. B 63, 144502 (2001).

${ }^{10}$ Yan Chen, Z. D. Wang, J. X. Zhu, and C. S. Ting, Phys. Rev. Lett. 89, 217001 (2002).

${ }^{11}$ B. Lake, G. Aeppli, K. N. Clausen, D. F. McMorrow, K. Lefmann, N. E. Hussey, N. Mangkorntong, M. Nohara, H. Takagi, T. E. Mason, and A. Schröder, Science 291, 1759 (2001); B. Lake, H. M. Rønnow, N. B. Christensen, G. Aeppli, K. Lefmann, D. F. McMorrow, P. Vorderwisch, P. Smeibidl, N. Mangkorntong, T. Sasagawa, M. Nohara, H. Takagi, and T. E. Mason, Nature (London) 415, 299 (2002).

${ }^{12}$ J. E. Hoffman, E. W. Hudson, K. M. Lang, V. Madhavan, S. H. Pan, H. Eisaki, S. Uchida, and J. C. Davis, Science 295, 466 (2002).

${ }^{13}$ S. J. Hagen, C. J. Lobb, R. L. Greene, M. G. Forrester, and J. H. Kang, Phys. Rev. B 41, 11630 (1990).

${ }^{14}$ Y. Aharonov and A. Casher, Phys. Rev. Lett. 53, 319 (1984).

${ }^{15}$ Y. Aharonov and D. Bohm, Phys. Rev. 115, 485 (1959).

${ }^{16}$ A. L. Fetter and J. D. Walecka, Quantum Theory of Many-
Particle Systems (Dover, New York, 2003).

${ }^{17}$ Zhou Shi-Ping, Chin. Phys. 10, 541 (2001).

${ }^{18}$ S. Daul, D. J. Scalapino, and S. R. White, Phys. Rev. Lett. 84, 4188 (2000).

${ }^{19}$ M. A. Kastner, R. J. Birgeneau, G. Shirane, and Y. Endoh, Rev. Mod. Phys. 70, 897 (1998).

${ }^{20}$ V. F. Mitrovic, E. E. Sigmund, M. Eschrig, H. N. Bachman, W. P. Halperin, A. P. Reyes, P. Kuhns, and W. G. Moulton, Nature (London) 413, 505 (2001); V. F. Mitrovic, E. E. Sigmund, W. P. Halperin, A. P. Reyes, P. Kuhns, and W. G. Moulton, Phys. Rev. B 67, 220503(R) (2003).

${ }^{21}$ T. Hanaguri, C. Lupien, Y. Kohsaka, D.-H. Lee, M. Azuma, M. Takano, H. Takagi, and J. C. Davis, Nature (London) 430, 1001 (2004).

${ }^{22}$ K. McElroy, D.-H. Lee, J. E. Hoffman, K. M. Lang, J. Lee, E. W. Hudson, H. Eisaki, S. Uchida, and J. C. Davis, Phys. Rev. Lett. 94, 197005 (2005).

${ }^{23}$ M. Civelli, M. Capone, S. S. Kancharla, O. Parcollet, and G. Kotliar, Phys. Rev. Lett. 95, 106402 (2005).

${ }^{24}$ G. Seibold, J. Lorenzana, and M. Grilli, Phys. Rev. B 75, 100505(R) (2007).

${ }^{25}$ N. W. Ashcroft and N. D. Mermin, Solid State Physics (Saunders, Philadephia, 1976).

${ }^{26}$ Shi-Ping Zhou, Yao-Ming Shi, Bao-He Zhu, and Guo-Qiao Zha, Phys. Rev. B 73, 174503 (2006).

${ }^{27}$ Bao-He Zhu, Shi-Ping Zhou, Yao-Ming Shi, Guo-Qiao Zha, and Kui Yang, Phys. Rev. B 74, 014501 (2006).

${ }^{28}$ Hans Hilgenkamp, Ariando, Henk-Jan H. Smilde, Dave H. A. Blank, Guus Rijnders, Horst Rogalla, John R. Kirtley, and Chang C. Tsuei, Nature (London) 422, 50 (2003).

${ }^{29}$ Robert H. Silsbee, J. Phys.: Condens. Matter 16, R179 (2004).

${ }^{30} \mathrm{~S}$. O. Valenzuela and M. Tinkham, Nature (London) 442, 176 (2006).

${ }^{31}$ A. L. Fetter, Phys. Rev. 140, A1921 (1965). 